\title{
Pancreatic cancer: a therapeutic challenge yet to be met
}

\author{
Jane de Lartigue, PhD
}

A

lthough there are numerous hard-to-treat tumor types, pancreatic cancer, which most commonly presents as pancreatic ductal adenocarcinoma (PDA) is particularly notorious and arguably the most challenging and deadly of all cancers. It is currently ranked as the fourth most common cause of cancer-related mortality, and looks set to move up to the number 2 slot within the next 15 years. ${ }^{1}$ Here, we discuss the evolution of much-needed novel treatment strategies.

\section{A dismal prognosis}

While the prognosis for many cancers has been steadily improving thanks to better screening or treatment, barely a dent has been made in the 5-year survival rates for patients with PDA in the past 3 decades, which remain stubbornly around 5\%. . $^{2,3}$

There are a number of factors that contribute to this dismal prognosis. Because the disease often does not present early-stage symptoms and there are no effective screening tests, more than half of all patients are diagnosed with advanced disease and median survival is less than a year. Roughly another third of patients are diagnosed with locally advanced disease that is not amenable to surgical resection and survival times are only marginally better. Even among patients who are diagnosed early and who have resectable disease, recurrence occurs often and median survival averages about 2 years. ${ }^{4}$

Furthermore, PDA is highly aggressive and resistant to most forms of treatment. In large part, this may be owing to another challenging aspect of pancreatic cancer biology - the unique tumor microenvironment. Pancreatic tumors are often surrounded by a massive growth of dense fibrous tissue that can impair the delivery of drugs at an effective concentration..$^{5-7}$

More recently, researchers have put forth a hypothesis that pancreatic cancer may actually develop as a systemic disease. There is evidence of metastatic spread of disease even before a pancreatic tumor becomes histologically evident. ${ }^{8,9}$ Proponents of this theory argue that we will only begin to make progress in the treatment of pancreatic tumors when we address them with effective systemic therapies. This may partly explain why chemotherapy is the only treatment to date that has proven effective in prolonging survival.

\section{Survival gains with chemotherapy advances}

Since the late 1990s, the standard of care for patients with pancreatic cancer has been the chemotherapeutic agent gemcitabine. A significant breakthrough for patients with advanced disease in the past 5 years has been the addition of 3 other chemotherapy regimens that have provided improvements in survival and have largely replaced gemcitabine monotherapy, though at the cost of increased toxicity so that their use is limited to patients with good performance status.

FOLFIRINOX is a combination chemotherapy containing the drugs fluorouracil, leucovorin, irinotecan, and oxaliplatin and was the first drug tested in advanced pancreatic cancer that pushed median survival past 1 year. The second regimen combines gemcitabine with nab-paclitaxel, a novel nanoparticle albumin-bound formulation of paclitaxel with improved pharmacokinetic properties. ${ }^{10,11}$

Most recently, the US Food and Drug Administration (FDA) approved a nanoliposomal formulation of irinotecan, in which the chemotherapy drug is encapsulated in a nanoliposomal construct that shields it from activation until it reaches the tumor, increasing anti-tumor efficacy while limiting systemic toxicity. ${ }^{12}$ These chemotherapeutic agents are also being investigated in the locally advanced and adjuvant treatment settings.

\section{Targeted therapies miss their mark}

Therapeutic advances in other tumor types have often come from a more comprehensive understanding of the molecular underpinnings of the disease. Genome-sequencing studies have revealed more than 60 different types of genomic alterations in PDA, including 4 major driver mutations (Figure

JCSO 2016;14(12):531-536. (2016 Frontline Medical Communications. doi: 10.12788/jcso.0312. 
TABLE Ongoing clinical trials in pancreatic cancer

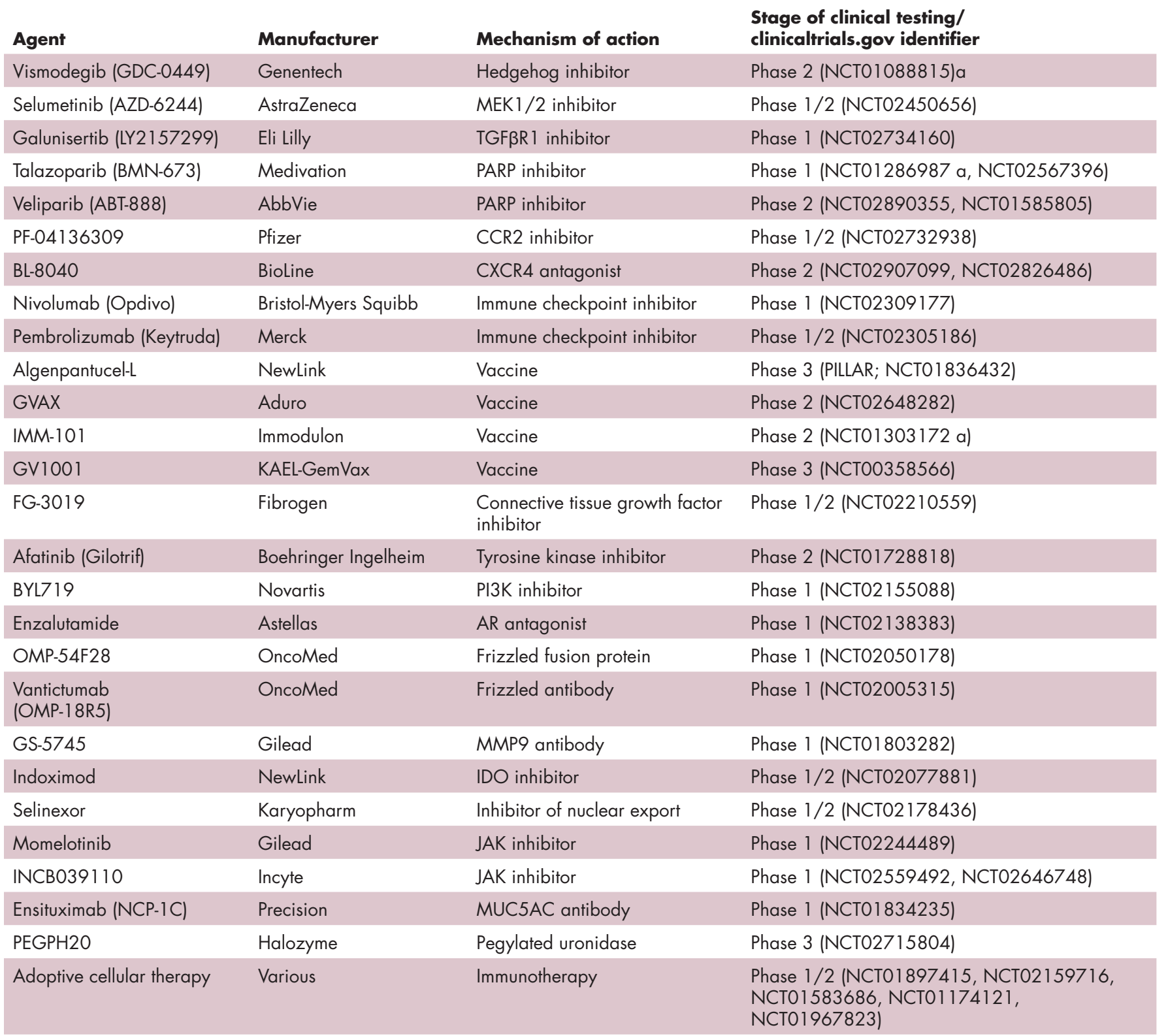

AR, androgen receptor; CCR2, C-C chemokine receptor type 2; CXCR4, C-X-C chemokine receptor type 4; IDO, indoleamine 2,3-dioxygenase; JAK, janus kinase; MEK, mitogen-activated protein kinase kinase; MMP-9, matrix metalloproteinase-9; MUC5AC, mucin 5AC; TGF $\beta R$ 1, transforming growth factor beta receptor 1; PI3K, phosphatidylinositol-3-kinase; PARP, poly(ADP) ribose polymerase;

angoing, but not actively recruiting participants.

1): KRAS and $C D K N 2 A$, which are almost ubiquitously observed in pancreatic cancer patients; TP53, in 75\%$90 \%$ of patients; and SMAD4, in about half of patients. At least 12 core signaling pathways have been repeatedly implicated in PDA development and progression, including DNA repair, apoptosis, cell-cycle control, embryonic development, and others. ${ }^{13,14}$

Although myriad targets have been tested and many have shown significant potential, almost invariably they fail to provide survival benefits. The exception is the epidermal growth factor receptor (EGFR) inhibitor erlotinib. After study findings suggested that the EGFR was overexpressed in up to $90 \%$ of pancreatic cancers, the EGFR became a heavily investigated target. For the most part studies of EGFR inhibitors were disappointing, however, erlotinib was approved by the FDA in combination with 


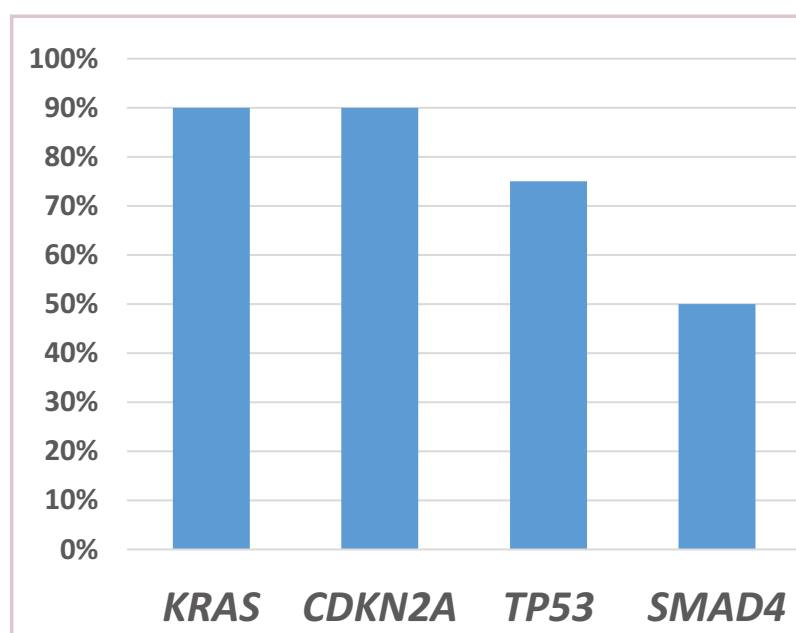

FIGURE 1 Genome sequencing studies of pancreatic adenocarcinoma have revealed four major genetic drivers. As in other cancers, major research efforts have focused on attempting to target these drivers for therapeutic purposes, with limited success to date.

gemcitabine and remains the only approved targeted therapy for pancreatic cancer.

Whether erlotinib really provides a survival benefit remains hotly contested, and any advantage comes at a cost of increased skin and gastrointestinal toxicity, so it is not widely used. EGFR inhibitors have been tested only in unselected patients, and the search for predictive biomarkers of response continues in the hope that this might enable clinicians to harness the full benefit of these drugs. ${ }^{15,16}$

Besides EGFR, other high-profile targets in pancreatic cancer include $K R A S$, as one of the most prolific genetic mutations. Unfortunately, direct targeting of the mutant KRAS protein has proven challenging for a number of reasons. However, numerous attempts have been made at indirect targeting; for example, farnesylation is a key posttranslational modification of RAS, required for its activation, and inhibitors of the farnesyl transferase enzyme have been tested in pancreatic cancer. However, this has not proven to be a successful therapeutic strategy, and efforts to find novel ways of targeting RAS continue. ${ }^{17}$

Another possibility for therapeutically disrupting RASmutant cancers is to target downstream signaling components. Numerous different targets have been explored, including the phosphatidylinositol-3-kinase (PI3K) and mammalian target of rapamycin (mTOR) proteins and components of the mitogen-activated protein kinase (MAPK) pathway, which is also activated by RAS. ${ }^{18}$ Although inhibitors of these proteins have had success in other tumor types, that success has not been translated into any clinical benefit in pancreatic cancer to date. Clinical trials are ongoing, including of the PI3K inhibitor, BYL-719, and the mitogen-activated protein kinase kinase (MEK) inhibitor, selumetinib.
Among the cellular processes that have been implicated in the development and progression of pancreatic cancer, DNA repair has recently been in the spotlight. About $10 \%$ of patients with pancreatic cancer have somatic and germline mutations in the breast cancer susceptibility genes, $B R C A 1$ and $B R C A 2$, and another $12 \%$ may have mutations that confer "BRCAness," in other words, the impact of these mutations on DNA repair processes is similar to those that result from $B R C A 1 / 2$ mutations. BRCAness is potentially an important property as it may indicate sensitivity to the poly(ADP)ribose polymerase (PARP) inhibitor class of drugs.

The PARP enzymes are involved in the repair of single-strand breaks (SSBs) in the DNA that can arise from environmental impact or from defects in DNA replication. PARP inhibition prevents SSB repair and the result is the development of a double-strand break (DSB) in the DNA, which can be lethal to the cell if it is not repaired.

Proteins such as BRCA1 and BRCA2 play a role in the homologous recombination (HR) pathway of DNA repair that is responsible for fixing DSBs. Thus, these proteins repair the damage caused by PARP inhibitors, so cancer cells with $B R C A 1 / 2$ mutations or similar mutations that result in dysfunctional $\mathrm{HR}$ are exquisitely sensitive to PARP inhibition. ${ }^{19,4}$

Trials of PARP inhibitors in pancreatic cancer patients are ongoing and is one of the most promising molecularly targeted strategies currently being evaluated. Talazoparib and veliparib are being investigated in numerous phase 1 and phase 2 trials, respectively (Table 1 ).

\section{Tumor microenvironment offers new targets}

In recent years, it has become increasingly clear that the tumor microenvironment, the non-cancerous cells and their connective tissue, as well as the vasculature and lymph vessels that infiltrate the space surrounding the cancerous mass, have a vitally important role in the establishment and maintenance of a tumor and resistance to anti-cancer therapy.

The tumor microenvironment can act as a physical barrier to the delivery of anti-cancer drugs, preventing them from reaching the target cancer cells at a therapeutically active concentration. There is also evidence for cross-talk between the malignant cells and the cells of the microenvironment via which the cancer cells are able to promote the optimal environment for growth, proliferation, survival and resistance to therapy. ${ }^{20,21}$

As a result, the tumor microenvironment has become a hot target for therapeutic manipulation. In pancreatic cancer, the focus has been on blocking the cell signaling pathways that are activated in the non-cancerous cells of the microenvironment that play a role in creating an oncogenic niche.

The Hedgehog, Notch, and Wnt signaling pathways, 


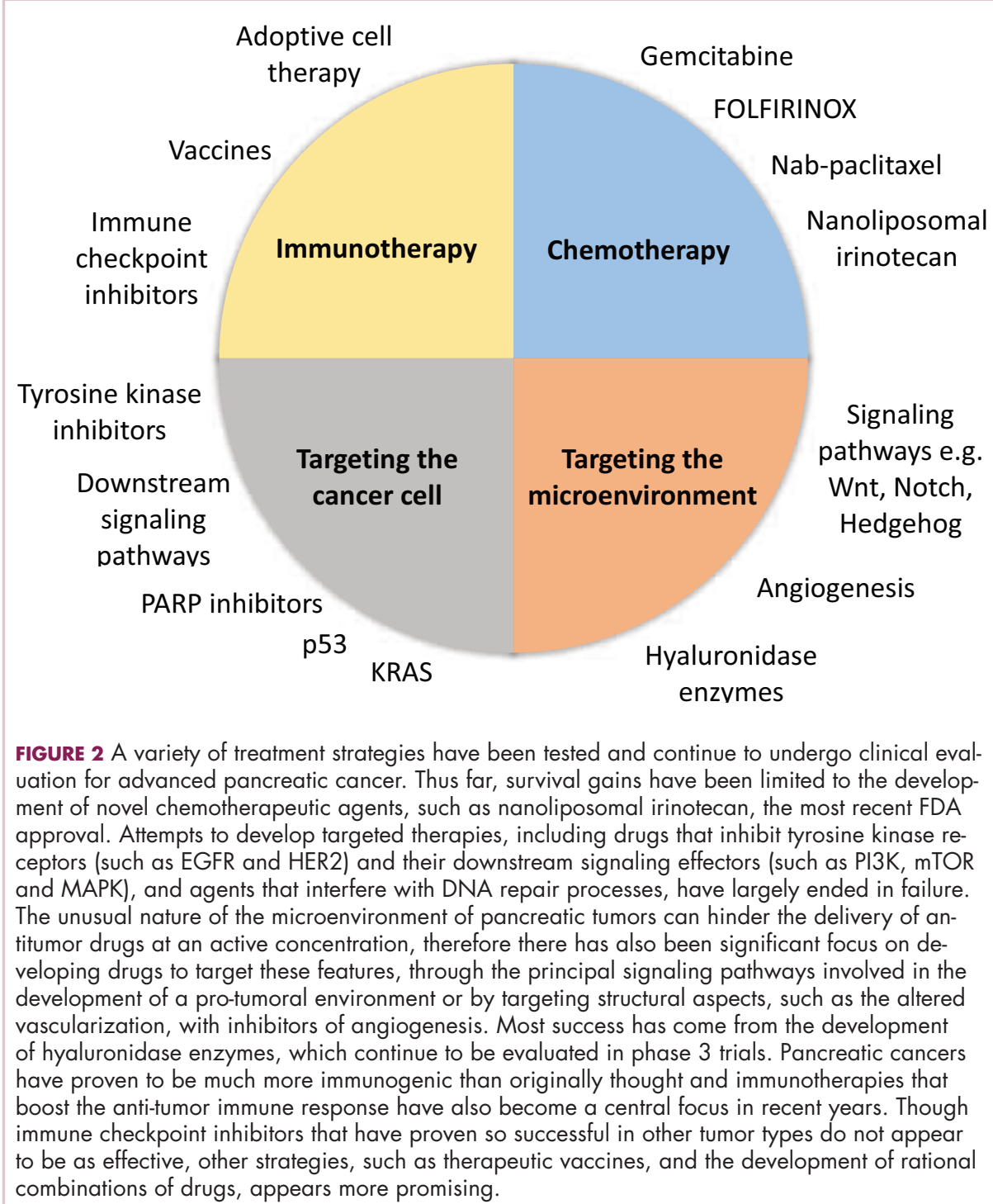

ronment is to try to overcome the physical barrier it can present. One approach that is currently undergoing evaluation in pancreatic cancer is to deplete the levels of hyaluronic acid (HA) in the tumor microenvironment. HA is a glycosaminoglycan that is one of the central components of the extracellular matrix and is also thought to be abundant in the desmoplastic reaction - the dense growth of fibrous tissue - that surrounds pancreatic tumors. ${ }^{24,25}$

PEGPH20, a pegylated form of the hyaluronidase enzyme that depletes HA levels, is being developed and could help break down this physical barrier. This is currently one of the most promising therapeutic avenues in the pancreatic cancer, with numerous clinical trials ongoing, including a phase 3 study in combination with nab-paclitaxel and gemcitabine in patients with previously untreated disease.

\section{Promise of immunotherapy requires clarification}

In lieu of targeted therapies that directly attack the tumor, the concept of stimulating an anti-tumor immune response to indirectly kill cancer cells has been gaining traction in the past decade. The major focus has been on developing drugs that activate cytotoxic $\mathrm{T}$ cells, the central mediators of the adaptive immune response. Drugs that block the T-cell inhibitory molecular pathways that are exploited by cancer cells to

all vital to embryonic development, are also thought to be highly dysregulated in the stromal cells in the pancreatic tumor microenvironment. It's thought that cancer cells activate these pathways in the stromal cells by producing their respective ligands, in other words the pathway is activated in a paracrine manner. These pathways may also be involved in the maintenance of stem cell-like properties in some pancreatic cancer cells. $18,7,22$

However, small-molecule inhibitors of these pathways have demonstrated limited efficacy in patients with pancreatic cancer. At least in part, this may be owing to poor delivery to their site of action, but recent study findings have also suggested vastly more complex roles for these signaling pathways in pancreatic cancer and that they may be involved in both cancer progression and prevention. ${ }^{23}$

Another possibility for targeting the tumor microenvi- downregulate the immune response mounted against them - the so-called immune checkpoint inhibitors - have had a ground-breaking impact in several tumor types.

The success of the immune checkpoint inhibitors has not been recapitulated in pancreatic cancer, at least not in the case of monotherapy. However, ongoing clinical trials have shifted their focus to testing potentially synergistic combinations with checkpoint inhibitors, such as with other types of immunotherapy and with drugs targeting the tumor microenvironment. ${ }^{26,27} \mathrm{BL}-8040$, an inhibitor of the $\mathrm{C}-\mathrm{X}-\mathrm{C}$ chemokine receptor type 4 (CXCR4), which plays a part in the cross-talk between the tumor and the stroma, in addition to numerous other physiological processes implicated in the development and progression of cancer, is being evaluated in a phase 2 trial in combination with the immune checkpoint inhibitor pembrolizumab in metastatic pancreatic cancer (KEYNOTE-202; NCT02826486). 
Alternative ways of targeting the $\mathrm{T}$ cells and other cells of the immune system are also being tested in pancreatic cancer. Indoleamine-2,3-dioxygenase (IDO) is an enzyme involved in breaking down the essential amino acid tryptophan and has immunomodulatory effects because the tryptophan metabolites that are subsequently generated are toxic to $\mathrm{T}$ cells. Furthermore, IDO directly activates the regulatory $\mathrm{T}$ cells, which have immunosuppressive functions. IDO inhibitors have been developed in the hope that blocking the effects of IDO could shift the balance of cytotoxic versus regulatory $\mathrm{T}$ cells in favor of the former. Overexpression of IDO has been noted in pancreatic tumors and clinical trials of the IDO inhibitor indoximod are ongoing. ${ }^{28}$

To date, the most promising immunotherapeutic strategy in pancreatic cancer has been therapeutic vaccines. Algenpantucel-L is a whole-cell vaccine that showed significant promise in phase 2 trials. ${ }^{29}$ The highly anticipated phase 3 IMPRESS trial evaluated the addition of this vaccine to standard of care in patients with resected disease. Unfortunately, the investigators recently reported that the trial failed to meet its primary endpoint of improved overall survival (OS). ${ }^{30}$ The phase 3 PILLAR trial of algenpantucel- $\mathrm{L}$ in combination with standard of care in patients with borderline resectable/locally advanced disease is ongoing, but not recruiting patients (NCT01836432).

Two other vaccines showed significant promise; GVAX, also a whole cell vaccine, and CRS-207, a weakened form of the Listeria monocytogenes bacterium, modified to secrete mesothelin, a tumor cell antigen. As a combination strategy, they were awarded Breakthrough Therapy designation from the FDA in 2014, but a recent phase 2 a study failed to meet its primary endpoint, and patients in the combination arm actually did worse than those treated with chemotherapy or GVAX alone. ${ }^{31}$ GVAX continues to be evaluated separately, in several phase 2 trials in combination with other therapies.

IMM-101 is another whole-cell vaccine that contains heat-killed Mycobacterium obuense. The results of a phase 2 trial in which it was combined with gemcitabine and compared to gemcitabine alone were recently reported. In the overall population, median OS was 6.7 months in the combination arm, compared with 5.6 months in the gemcitabine arm. Although this difference in OS was not statistically significant, in a predefined metastatic subgroup, OS was significantly improved from 4.4 months to 7 months with the combination (hazard ratio, $0.54 ; P=.01$ ). ${ }^{32}$

\section{References}

1. Rahib L, Smith BD, Aizenberg R, et al. Projecting cancer incidence and deaths to 2030: the unexpected burden of thyroid, liver and pancreas cancers in the United States. Cancer Res. 2014;74(11):2913-2921.

2. Siegel R, Miller KD, Jemal A. Cancer statistics, 2015. CA Cancer J Clin. 2015;65:5-29.

3. Jemal A, Bray F, Center MM, et al. Global cancer statistics. CA Cancer J Clin. 2011;61(2):69-90.

4. Akinleye A, Iragavarapu C, Furqan M, et al. Novel agents for advanced pancreatic cancer. Oncotarget. 2015;6(37):39521-39537.

5. Apte MV, Park S, Phillips PA, et al. Desmoplastic reaction in pancreatic cancer: role of pancreatic stellate cells. Pancreas. 2004;29:179-187.

6. Pandol S, Edderkaoui M, Gudovsky I, et al. Desmoplasia of pancreatic ductal adenocarcinoma. Clin Gasteroenterol Hepatol. 2009;7:S44-S47

7. Garrido-Laguna I and Hidalgo M. Pancreatic cancer: from stateof-the-art treatments to promising novel therapies. Nat Rev Clin Oncol. 2015;12:319-334.

8. Rhim AD, Mirek ET, Aiello NM, et al. EMT and dissemination precede pancreatic tumor formation. Cell. 2012;148:349-361.

9. Sohal DP, Walsh RM, Ramanathan RK, et al. Pancreatic adenocarcinoma: treating a systemic disease with systemic therapy. J Natl Cancer Inst. 2014;106(3):dju011.

10. Conroy T, Desseigne F, Ychou M, et al. FOLFIRINOX versus gemcitabine for metastatic pancreatic cancer. $\mathrm{N}$ Engl J Med. 2011;364:1817-1825.

11. Von Hoff DD, Ervin T, Arena FP, et al. Increased survival in pancreatic cancer with nab-paclitaxel plus gemcitabine. N Engl J Med. 2013;369:1691-1703.

12. Wang-Gillam A, Li C-P, Bodoky G, et al. Nanoliposomal irinotecan with fluorouracil and folinic acid in metastatic pancreatic cancer after previous gemcitabine-based therapy (NAPOLI-1): a global, randomised, open-label, phase 3 trial. Lancet. 2016;387(10018):545-557.

13. Wood LD, Hruban RH. Pathology and molecular genetics of pan-

creatic neoplasms. Cancer J. 2012;18:492-501.

14. Jones S, Zhang X, Parsons DW, et al. Core signaling pathways in human pancreatic cancers revealed by global genomic analyses. Science. 2008;321:1801-1806.

15. Moore MJ, Goldstein D, Hamm J, et al. Erlotinib plus gemcitabine compared with gemcitabine alone in patients with advanced pancreatic cancer: a phase III trial of the National Cancer Institute of Canada Clinical Trials Group. J Clin Oncol. 2007;25:1960-1966.

16. Kelley RK and Ko AH. Erlotinib in the treatment of advanced pancreatic cancer. Biologics. 2008;2(1):83-95.

17. Zeitouni D, Pylayeva-Gupta Y, Der CJ, Bryan JL. KRAS mutant pancreatic cancer: no lone path to an effective treatment. Cancers. 2016;8(4):45-67.

18. Karanikas M, Esempidis A, Chasan Z, et al. Pancreatic cancer from molecular pathways to treatment opinion. J Cancer. 2016;7(10):1328-1339.

19. Bhalla A and Saif MW. PARP inhibitors in BRCA-associated pancreatic cancer. J Oncol Prac. 2014;15(4):340-343.

20. Fang H, DeClerck YA. Targeting the tumor microenvironment: From understanding pathways to effective clinical trials. Cancer Res. 2013;73(16):4965-4977.

21. Chen F, Zhuang X, Lin L, et al. New horizons in tumor microenvironment biology: challenges and opportunities. BMC Medicine. 2015;13:45.

22. Vaz AP, Ponnusamy MP, Seshacharyulu P, Batra SK. A concise review on the current understanding of pancreatic cancer stem cells. J Cancer Stem Cell Res. 2014;2:e1004.

23. Berndt JD. Complex roles for hedgehog signaling in pancreatic cancer. Sci Signal. 2014;7(337):ec204.

24. Provenzano PP, Hingorani SR. Hyaluronan, fluid pressure, and stromal resistance in pancreas cancer. Gut. 2011;60(6):861-868.

25. Provenzano PP, Cuevas C, Chang AE, et al. Enzymatic targeting of the stroma ablates physical barriers to treatment of pancreatic ductal adenocarcinoma. Cancer Cell. 2012;21(3):418-429.

26. Kunk PR, Bauer TW, Slingluff CL, Rahma OE. From bench to bedside a comprehensive review of pancreatic cancer immunother- 


\section{Feature}

apy. J Immunother Cancer. 2016;4:14.

27. Lutz ER, Kinkead H, Jaffee EM, Zheng L. Priming the pancreatic cancer tumor microenvironment for checkpoint immunotherapy. OncoImmunol. 2014;3(11):e962401.

28. Moon YW, Hajjar J, Hwu P and Naing A. Targeting the indoleamine 2,3-dioxygenase pathway in cancer. J Immunother Cancer. 2015;3:51.

29. Hardacre JM, Mulcahy M, Small W, et al. Addition of algenpantucel-L immunotherapy to standard adjuvant therapy for pancreatic cancer: a phase 2 study. J Gastrointest Surg. 2013;17(1):94-100.

30. NewLink Genetics. NewLink Genetics announces results from phase 3 IMPRESS trial of algenpantucel-L for patients with resected pancreatic cancer. http://investors.linkp.com/releasedetail. cfm?releaseid=969978. Published May 9, 2016. Accessed October 25, 2016.

31. Le DT, Wang-Gillam A, Picozzi V Jr, et al. A phase 2, randomized trial of GVAX Pancreas and CRS-207 immunotherapy versus GVAX alone in patients with metastatic pancreatic adenocarcinoma: Updated results [ASCO Gastrointestinal Cancers Symposium abstract 177]. J Clin Onco. 2014;32(suppl 3).

32. Dalgleish AG, Stebbing J, Adamson DJA, et al. Randomised, openlabel, phase II study of gemcitabine with and without IMM-101 for advanced pancreatic cancer. Br J Cancer. 2016;115:789-796 